\title{
Lipophilic Gold Grating for SERS Detection of Biological Objects ${ }^{\dagger}$
}

\author{
O. Guselnikova ${ }^{1,2, *}$, K. Hrobonova ${ }^{2}$, P. Postnikov ${ }^{2, *}$, O. Lyutakov ${ }^{1,2, *}$ and V. Svorcik ${ }^{2}$ \\ 1 University of Chemical Technology, Prague, Czech Republic \\ 2 Tomsk Polytechnic University, Tomsk, Russia; karoli.f@seznam.cz (K.H.); svorcikv@vscht.cz (V.S.) \\ * Correspondence: guselnikovaoa@tpu.ru (O.G.); postnikov@tpu.ru (P.P.); lyutakoo@vscht.cz (O.L.) \\ + Presented at the Eurosensors 2017 Conference, Paris, France, 3-6 September 2017.
}

Published: 4 August 2017

\begin{abstract}
We introduce label-free plasmon-active platform based on ordered gold gratings with the covalently modified surface for detection of lipophilic bioobjects. Alkylbenzene groups with different chain length $\left(\mathrm{CH}_{3}-, \mathrm{C}_{4} \mathrm{H}_{9-}, \mathrm{C}_{10} \mathrm{H}_{21}-\right.$ and $\left.\mathrm{C}_{16} \mathrm{H}_{33}\right)$ were grafted spontaneously and electrochemically for improvement of affinity with lipophilic compounds. Modified grating surfaces were characterized by XPS, CVA, wettability, AFM and Raman spectroscopy techniques.
\end{abstract}

Keywords: SERS; sensors; diazonium salts; surface modification; cholesterol

\section{Introduction}

Early stage diagnosis and sensing is the one of the most essential issue for extremely important social problems. For instance, there are a number of molecules and biomarkers that may reveal the presence of a disease and their recognition is still challenging due to extremely low concentrations. Ultrasensitivity, selectivity and high speed of analysis provided a unique possibility to the application of plasmonics for the sensing of biomedical objects [1]. For effective detection, plasmonic substrates should be modified to improve the contact between the analyte and the plasmonic material, what allow increase the concentration of targeted chemical species on the surface [2]. Here, we offer the plasmon-active substrate for detection of lipophilic compounds based on the highly-ordered gold gratings. In order to improve affinity with biological lipophilic compounds, gold gratings surfaces were modified by arenediazonium tosylates (ADTs) with different length of aliphatic substituents $\left(\mathrm{CH}_{3-}, \mathrm{C}_{4} \mathrm{H}_{9-}, \mathrm{C}_{10} \mathrm{H}_{21}-\right.$ and $\left.\mathrm{C}_{16} \mathrm{H}_{33}\right)$ by spontaneous and electrochemical methods.

\section{Materials and Methods}

Gold grating were prepared according to [3]. The deposition of gold was accomplished from $\mathrm{Au}$ target (purity of $99.99 \%$, provided by Safina, Czech Republic).

4-methylbenzenediazonium tosylate $\left(\mathrm{ADT}-\mathrm{CH}_{3}\right)$, 4-butylbenzenediazonium tosylate $\left(\right.$ ADT- $\left.\mathrm{C}_{4} \mathrm{H}_{9}\right)$, 4-decylbenzenediazonium tosylate (ADT- $\left.\mathrm{C}_{10} \mathrm{H}_{21}\right)$, 4-hexadecylbenzenediazonium tosylate (ADT- $\mathrm{C}_{16} \mathrm{H}_{33}$ ) were prepared according to previously published procedure [4].

Alkylphenyl functional groups were grafted spontaneously by soaking of the freshly sputtered $\mathrm{Au}$ films in $1 \mathrm{mM}$ solutions of corresponding ADTsin deionized water for $10 \mathrm{~min}$. Electrografting of ADTs was performed in $1 \mathrm{mM}$ water solutions of corresponding ADTs under the potential $-2 \mathrm{~V}$ for $10 \mathrm{~min}$. After modification the metal substrates were rinsed under sonication sequentially with deionized water, ethanol, and acetone for $10 \mathrm{~min}$ and dried in desiccator for $3 \mathrm{~h}$. 


\section{Measurement Techniques}

The X-ray photoelectron spectroscopy (XPS) was performed using an Omicron Nanotechnology ESCAProbeP spectrometer fitted with monochromatic Al K Alpha X-ray source working at $1486.6 \mathrm{eV}$. For characterization of the sample surface and nanomechanical mapping before and after surface modification, the peak force AFM technique was applied using the Icon (Brucker) microscope. Raman scattering was measured on portable ProRaman-L spectrometer (Laser power $15 \mathrm{~mW}$ ) Raman spectrometer with $785 \mathrm{~nm}$ excitation wavelengths. Spectra were measured 30 times, each of them with $3 \mathrm{~s}$ accumulation time. Water contact angles were measured by goniometer Surface Energy Evaluation System (Masaryk University, Czech Republic) at 10 positions with distilled water at room temperature. Electrochemical characterization of gold surfaces was performed by cyclic voltammetry $(\mathrm{CV})$ at the potential range from $-100 \mathrm{mV}$ to $700 \mathrm{mV}$ with scan rate $50 \mathrm{mV} \mathrm{s}^{-1}$ using 1 $\mathrm{mM}$ potassium ferrocyanide (II) in $0.1 \mathrm{M}$ potassium nitrate as an aqueous medium and $1 \mathrm{mM}$ potassium ferrocyanide in $0.1 \mathrm{M}$ potassium nitrate with $50 \%$ of methanol $(v / v)$ as a mixed water-methanol medium.

\section{Results and Discussion}

Prepared gold gratings were chosen as a substrate for plasmonic sensor fabrication due to homogeneous response and strong signal enhancement, as was described before [5]. The surface of the gold was modified by a range of arenediazonium tosylates (ADTs): ADT- $\mathrm{CH}_{3}, \mathrm{ADT}-\mathrm{C}_{4} \mathrm{H}_{9}$, ADT- $\mathrm{C}_{10} \mathrm{H}_{21}$ and ADT- $\mathrm{C}_{16} \mathrm{H}_{33}$ in order to improve affinity with lipophilic biological compounds (Figure 1). Two modification approaches were tested: spontaneous and electrochemically induced grafting.

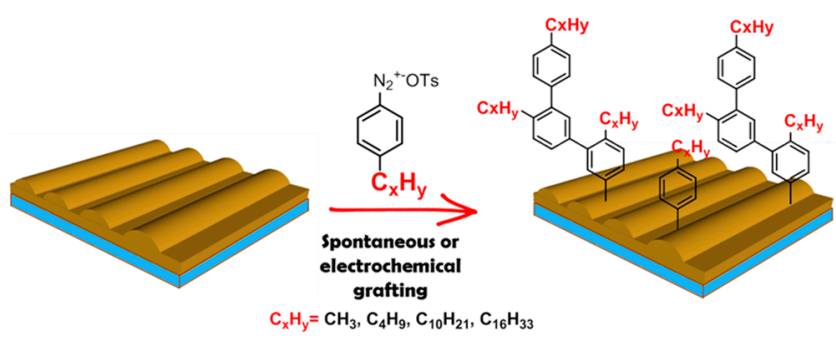

Figure 1. The scheme of the preparation of lipophilic gold gratings.

\subsection{Raman Spectroscopy}

To confirm the ADTs grafting, Raman spectroscopy was applied. Figure 2A,B show the spectra obtained after the spontaneous and electrochemically induced modification of the prepared gold grating surface. The measured intensity of spontaneously modified substrates (Figure 2A) was noticeably weaker than in case of electrochemically modified samples (Figure 2B), whose responses ranged in the order of hundreds. Results of Raman spectroscopy performed after the ADTs grafting indicate the presence of characteristic adsorption bands, vibrations of aromatic ring 1600-1640, 990$1090,510-710 \mathrm{~cm}^{-1}$ and vibrations of aliphatic chains $1355-1390,1050-1070,720-860 \mathrm{~cm}^{-1}$. 

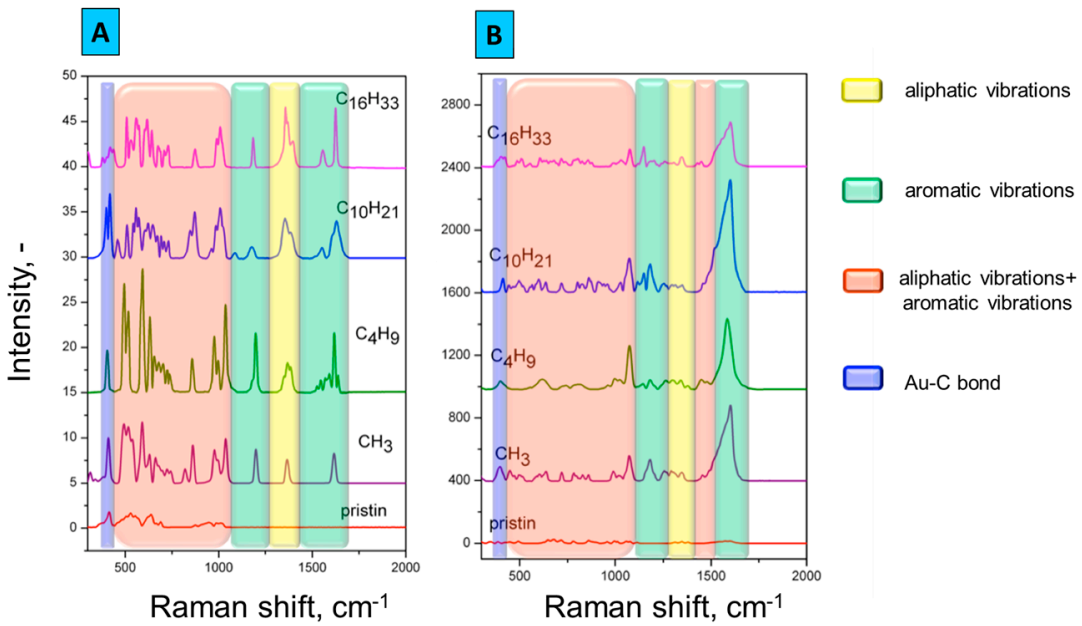

Figure 2. Raman spectra of gold gratings grafted by ADTs via spontaneous and electrochemical approaches.

\subsection{XPS}

The changes in surface elemental concentration after ADTs grafting was studied using XPS method (Table 1). After modification by both approaches, the C (1s) concentration increased, while $\mathrm{Au}(4 \mathrm{f})$ concentration almost disappeared due to formation of the thick organic layer. As it was expected with the increase of aliphatic chain length, the carbon concentration increased from 30.2 to $98.8 \%$. However, electrochemical modification led to a more pronounced increase in C concentration than in case of the spontaneous modification. In the case of electrochemical modification nitrogen $\mathrm{N}$ (1s) also appear on the surface. This can be explained by the formation of azo-bridges in the structure of grafted organic layer.

Table 1. Surface atomic element concentrations from XPS measured for pristine, grafted by ADTs.

\begin{tabular}{|c|c|c|c|c|c|}
\hline \multirow{2}{*}{ Sample } & \multicolumn{2}{|c|}{ Spontaneous } & \multicolumn{3}{|c|}{ Electrochemical } \\
\hline & C & $\mathrm{Au}$ & $\mathrm{C}$ & $\mathbf{N}$ & $\mathrm{Au}$ \\
\hline Pristine & 32.9 & 67.1 & 32.9 & - & 67.1 \\
\hline $\mathrm{CH}_{3}$ & 72.9 & 27.1 & 90.4 & 4.1 & 5.5 \\
\hline $\mathrm{C}_{4} \mathrm{H}_{9}$ & 83.7 & 16.3 & 97.7 & 2.2 & 0.1 \\
\hline $\mathrm{C}_{10} \mathrm{H}_{21}$ & 87.5 & 12.5 & 98.7 & 1.3 & - \\
\hline $\mathrm{C}_{16} \mathrm{H}_{33}$ & 92 & 8 & 98.8 & 1.2 & - \\
\hline
\end{tabular}

\subsection{Wettability}

Influence of ADTs grafting on surface wettability was investigated via measurement of water contact angle with the assumption that contact angles will increase with the increasing of aliphatic chain length in the structure of grafted ADTs. Results are presented in the Table 2 and it can be seen that electrochemically modified samples demonstrate a more rapid increase of surface hydrophobicity than spontaneous, which can be explained by the formation of thicker organic layer.

Table 2. Surface wettability (water contact angle) of pristine and grafted with ADTs gold gratings.

\begin{tabular}{ccc}
\hline & \multicolumn{2}{c}{ Contact Angle, $^{\circ}$} \\
\cline { 2 - 3 } & Spontaneous & Electrochemically \\
\hline Pristine & 82 & 82 \\
$\mathrm{CH}_{3}$ & 70 & 80 \\
$\mathrm{C}_{4} \mathrm{H}_{9}$ & 81 & 83 \\
$\mathrm{C}_{10} \mathrm{H}_{21}$ & 85 & 100 \\
$\mathrm{C}_{16} \mathrm{H}_{33}$ & 90 & 107 \\
\hline
\end{tabular}




\subsection{AFM}

The results of AFM measurements are shown in Figure 3A, where it can be noticed that modification by electrochemical mechanism led to more pronounced surface changes. It is also apparent that the grating surface morphology is influencr4d by ADTs grafting: the grating amplitude decreased with increasing of aliphatic chains length, the periodic structure becomes less noticeable especially in the case of relectro-chemical procedure. This phenomenon can be associated with the formation of polyphenylene layers, which particularly disguise the pristine surface features.
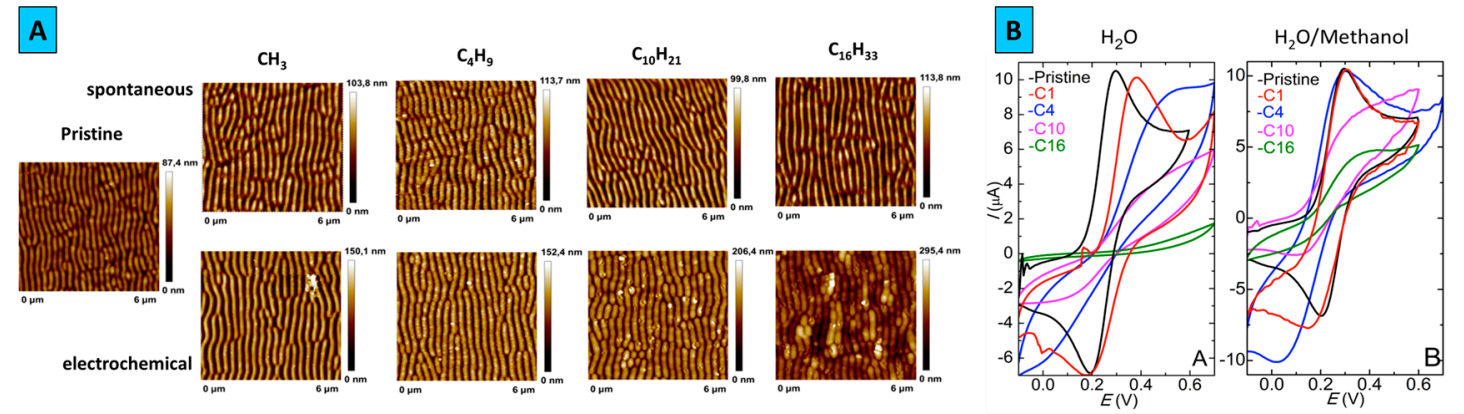

Figure 3. (A) Surface topography of pristine and modified by ADTs surfaces, (B) Cyclic voltamperometry of pristine and electrochemically modified samples.

\section{5. $C V A$}

The surface blocking induced by grafting procedure was examined by cyclic voltammetry, using the solution of potassium ferrocyanide with water or water/methanol mix as solvents (Figure 3B). In aqueous media with the increase of aliphatic chain length the oxidation/reduction peaks become less pronounced and the electron transfer kinetics is significantly suppressed by surface blocking. Obvious changes in cyclic voltammograms and slower electron transfer kinetics confirmed that ADTs block almost the entire surface of the gold grating. Similar electrochemical behavior was observed during the measurements in the mixed aqueous-methanol medium (50\% v/v). However, increase of carbon chain length did not lead to full peak suppression, indicating the influence of the used solvent.

\section{Conclusions}

The development of functional plasmonic-active substrates with hydrophobic surface for potential detection of lipophilic bioobjects was proposed. As plasmonic background, the periodical gold grating was created by the excimer laser pattering of polymer surface and further metal covering. For efficient interaction with lipophilic compounds, the gold surface was functionalized by two approaches: spontaneous and electrochemical grafting of arenedeiazonim tosylates with different alkyl chain lengths substituents. The grafting of organic functional groups was confirmed by Raman spectroscopy, XPS, wettability measurements, AFM and CVA. According to obtained results electrochemical approach will be more suitable for detection of lipophilic compounds due to higher amount of grafted alkylphenyl groups. Prepared functionalized SERS-active substrates can potentially applied for detection of various bioobjects and disease markers, such as cholesterol markers, lipophilic bacteria, lysozymes and cell membrane receptors, as well as polycyclic aromatic compounds, azodyes and polychlorinated aromatic compounds.

Acknowledgments: This work was supported by Tomsk polytechnic university (Project VIU-316/2017).

Conflicts of Interest: The authors declare no conflict of interest. 


\section{References}

1. Kumar, A.; Kim, S.; Nam, J.-M. Plasmonically Engineered Nanoprobes for Biomedical Applications. J. Am. Chem. Soc. 2017, 138, 14509-14525.

2. Alvarez-Puebla, R.A.; Liz-Marzan, L.M. Traps and cages for universal SERS detection. Chem. Soc. Rev. 2012, 41, 43-51.

3. Kalachyova, Y.; Mares, D.; Jerabek, V.; Zaruba, K.; Ulbrich, P.; Lapcak, L.; Svorcik, V.; Lyutakov, O. The Effect of Silver Grating and Nanoparticles Grafting for LSP-SPP Coupling and SERS Response Intensification. J. Phys. Chem. C 2016, 120, 10569-10577.

4. Filimonov, V.D.; Trusova, M.E.; Postnikov, P.S.; Krasnokutskaya, A.E.; Lee, Y.M.; Hwang, H.Y.; Kim, H.; Chi, K.-W. Unusually Stable, Versatile, and Pure Arenediazonium Tosylates: Their Preparation, Structures, and Synthetic Applicability. Org. Lett. 2008, 18, 3961-3964.

5. Guselnikova, O.; Postnikov, P.; Kalachyova, Y.; Kolska, Z.; Libansky, M.; Zima, J.; Svorcik, V.; Lyutakov, O. Large-Scale, Ultrasensitive, Highly Reproducible and Reusable Smart SERS Platform Based on PNIPAm-Grafted Gold Grating. ChemNanoMat 2017, 3, 135-144.

(C) 2017 by the authors. Licensee MDPI, Basel, Switzerland. This article is an open access article distributed under the terms and conditions of the Creative Commons Attribution (CC BY) license (http://creativecommons.org/licenses/by/4.0/). 